Review Article

\title{
Renal transplantation anesthetic experience of I 34 cases april 2000- april 2005
}

\begin{abstract}
Transplantation provides a near normal life and excellent rehabilitation compared to dialysis and is the preferred method of treatment for end stage renal disease patients. We describe our experience through a retrospective analysis of anesthesia management of 134 of living donors renal transplantation conducted between April 2000 - April 2005 at Ahmed Gasim hospital. Areas of interest include preoperative patient status, fluid management, hemodynamic satiability, anesthesia management and perioperative complications.

Recent advances in surgical technique; anesthesia management and immunosuppressive drugs have made renal transplantation safe and predictable. Preoperative patient optimization, intraoperative physiological stability and postoperative care of renal transplant patients have contributed to success of renal transplant program in our hospital.
\end{abstract}

Keywords: renal transplantation, end stage renal disease, general anesthesia
Volume 6 Issue I - 2016

\author{
Ahmed Ali Khalid Salih \\ Associate Professor of Anesthesia, Umm AL-qura University, \\ College of Applied Medical Sciences, Department of Anesthesia \\ Technology, Sudan
}

\begin{abstract}
Correspondence: Ahmed Ali Khalid Salih, Assistance Professor of Anesthesia, Umm AL-qura University College of Applied Medical Sciences, Department of Anesthesia Technology, MD and MBBS fellowship smb, Omdorman Military hospital, Sudan, Tel966583779886,Email astmr2005@gmail.com
\end{abstract}

Received: October 05, 2016 | Published: October 24, 2016

\section{Introduction}

Renal transplantation in the Sudan started in the early seventies in Khartoum antedating most of the countries in the middle east and Africa The program then collapsed along with everything else in the country due to economic recession.

The current program started in Ahmed Gasim Centre in April 2000. It is state sponsored. It depends on live related donors-usually the donor is a first degree relative of the recipient. There are no cadaveric donors. We had one twin donor, and the recipient is doing well, with a normally functioning graft. The warm Ischaemia time is essentially zero and cold ischaemia time is about 10 minutes, Urine production starts immediately-on table, If it doesn't, there is a surgical problem.

Organ viability associated with renal transplantation is a product of the managing of donor patient, the allograft, and the recipient patient short-and long- term outcome is influenced by perioperative fluid and drug treatment, and the function and viability of the transplanted kidney seem to be optimized if graft perfusion is maximized through mild hypervolemia. At the same time, careful balancing of intraoperative fluids is necessary against cardiovascular problems frequently encountered in patients with uremia. Close intraoperative monitoring, optimization of intravascular fluid volume status to maximize kidney perfusion and promote correction of electrolyte disturbances (especially potassium) are key to short and long term success of renal transplants.

We conducted a retrospective analysis of 134 cases of living renal transplants to identify the trends according to patient's age, sex, cause of chronic kidney disease, anesthesia management and the outcome of patient in our hospital.

\section{Anesthesia for renal transplantation review}

Chronically ill with conditions such as diabetes mellitus, hypertension, coronary artery disease and their complications. Most patients would have been initiated on either hemodialysis or peritoneal dialysis and present to the anesthesiologist with a long list of medications. The goal of this article is to review the perioperative management of patients undergoing kidney transplantation.

\section{Pre-operative considerations}

With advances in surgical and anesthetic techniques, older and more medically complex patients are being considered for renal transplantation. Most patients presenting to anesthesiologists for pre-surgical evaluation for transplantation would have had thorough medical evaluation and follow-up to optimize their comorbidities as part of routine preparation for transplantation. Absolute contraindications to transplantation include active infection, untreated malignancy, and predicted patient survival less than 5 years, risk of transplant graft loss greater than $50 \%$ at 1 year, inability to comply with immunosuppression regimen, and immunosuppression predicted to cause a life threatening complication. After a donor kidney becomes available, it is matched to a recipient as best as possible by blood cross matching, HLA typing, and testing donor T-cell against recipient serum.

\section{Cardiovascular}

The risk of cardiovascular disease is 10 to 30 times higher in dialysis patients than in the normal population and over $50 \%$ of deaths in dialysis patients result from cardiovascular disease. As a result, a careful evaluation of the cardiovascular system is required in patients being considered for transplantation. Special emphasis should be paid to the assessment of intravascular volume status, presence of hypertension, and anemia. A baseline 12 lead ECG should be obtained and resting transthoracic echocardiogram should be used to assess heart function for evidence of dilated cardiomyopathy and concentric hypertrophy which develop in response to chronically Patients waiting on the transplant list beyond one year, should be screened annually with 12 lead ECG and possibly resting TTE, although the usefulness of periodic screening for resting LV function while on the transplantation list is uncertain except in patients with moderate aortic stenosis who should be treated as rapid-progressors and therefore have yearly resting echocardiography. Recent studies have suggested that elevated pulmonary artery pressures are associated with adverse outcomes after renal transplantation .Renal transplant candidates found to have pulmonary hypertension on TTE should have right heart catheterization to confirm the diagnosis and full evaluation performed by a physician with expertise in pulmonary hypertension 
management. In summary, the goal of cardiovascular evaluation is to diagnose active or chronic CAD, determine the patient's functional status and optimize therapy prior to renal transplantation.

\section{Pulmonary}

The respiratory challenges facing the anesthesiologist during kidney transplantation surgery arise mostly from volume overload and pulmonary congestion. This usually results in hypoxemia and occasionally hypercapnia. In patients undergoing peritoneal dialysis, diaphragmatic splinting may lead to basal lung atelectasis and result in arterio-venous shunting .Dialysis and fluid removal prior to surgery is helpful with alleviating pulmonary congestion. There are no specific indications for further pulmonary work-up in kidney transplant patients unless the patient has other co morbid lung diseases.

\section{Gastrointestinal}

Uremia from kidney failure may lead to gastro paresis Additionally, many ESRD patients suffer from diabetes mellitus and autonomic neuropathy and should therefore be treated with full stomach precautions. Preoperative treatment with a Histamine2 blocker and metoclopramide are recommended. Hepatitis $\mathrm{C}$ virus (HCV) infection is associated with membranous nephropathy and membrano proliferative glomerulonephritis and is common among dialysis patients. In addition, patients on chronic hemodialysis are at an increased risk of acquiring $\mathrm{HCV}$ infection as a result of frequent blood transfuseons. HCV infection is a risk factor for death in renal transplantation patients who develop sepsis and septic shock.

\section{Renal and metabolic}

Since patients with ESRD are either on peritoneal dialysis or hemodialysis, they may present with electrolyte and metabolic abnormalities such as hyponatremia, hyperchloremia, hyperkalemia, hypocalcemia, hypermagnesemia and metabolic acidosis. The severity of these electrolyte derangements is usually related to the timing of the patient's last dialysis session. Perioperative hyperkalemia is most concerning in the perioperative period. Since these patients are usually chronically hyperkalemic, the ECG changes usually seen in hyperkalemia such as peaked T-waves, flat $\mathrm{P}$ waves, increased PR interval or widening QRS may not be present until much higher values are reached. Consequently, it is important to have periodic checks to detect hyperkalemia. To avoid complications of hyperkalemia, treatment with insulin, sodium bicarbonate, and beta-agonist should be considered if the potassium is greater than $5.5 \mathrm{mEq} / \mathrm{L}$. Routine preoperative electrolyte evaluation should be obtained prior to surgery.

\section{Hematologic}

Patient with ESRD usually presents with normochromic, normocytic anemia. Anemia has been linked to cardiovascular morbidity and mortality and Harnett et al. found the independent relative risk of mortality in dialysis patient to be 1.18 per $1.0 \mathrm{~g} / \mathrm{dl}$ decrease in hemoglobin level. Anemia in ESRD results from impaired erythropoiesis from decreased erythropoietin synthesis and release, and also decreased red blood cell life span. In addition, increased hemolysis, repeated blood loss during dialysis, uremia induced bone marrow suppression and iron, folate, vitamin B6/B12 deficiencies all contribute to anemia. Most patients are treated with synthetic erythropoietin to increase hemoglobin. The anemia is well tolerated in most patients due to compensatory increases in cardiac output, 2 , 3-DPG and a right ward shift of oxygen dissociation curve which results in improved tissue oxygenation.
The correction of decreased erythropoiesis, with erythropoietin, aids oxygen transport and decreases cardiac output, heart rate and cardiac workload, which leads to a decrease in left ventricular hypertrophy and overall improvement in cardiac status. Exercise capacity, cognitive and brain function and quality of life also improve. Ultimately, mortality is reduced. Patients may also be thrombocytopenic from uremia associated thrombocytopathia. A thorough history should be obtained to detect possible platelet dysfunction. Preoperative prothrombin time (PT), Partial thromboplastin time (PTT), INR, complete blood count $(\mathrm{CBC})$, and liver function tests should be obtained during the pretransplantation work-up.

\section{Endocrine}

Diabetes mellitus is the most common co-morbidity in patients with ESRD. Patients with diabetic nephropathy are known to have higher mortality rates than other patients with other causes of ESRD. The risk of cardiovascular disease is high, hence the need to screen for and treat coronary artery disease in diabetic patients undergoing transplantation. Good glycemic control is important before and during transplant and is associated with a lower mortality. Preoperatively, immediate point of care glucose should be checked prior to surgery. Glucose control between $120-200 \mathrm{mg} / \mathrm{dL}$ is recommended rather that tight glycemic control. Hyperphosphatemia resulting from decreased excretion of phosphate and hypocalcemia due to reduced absorption of calcium from the GI tract and vitamin D deficiency are very common. This leads to a secondary hyperparathyroidism and demineralization of bone possibly leading to pathologic fractures.

\section{Nervous system}

CNS manifestation such as malaise, fatigue, inability to concentrate, seizures and coma can be seen in patients who are uremic. These complications are reduced with dialysis. Other manifestations such as prior stroke may be seen due to the vascular disease that often accompanies ESRD. Peripheral and autonomic neuropathies can lead to orthostatic hypotension and silent myocardial ischemia.

\section{Intra-operative considerations}

Pharmacology: ESRD not only affects the pharmacokinetics and pharmacodynamics of drugs excreted by the kidneys, but also modifies the disposition of other drugs through changes in protein binding or hepatic metabolism. Hepatic drug metabolism is influenced either through induction or inhibition of hepatic enzymes or by alteration of hepatic blood flow, and the production and elimination of metabolites. Changes in body fluid distribution and circulatory volume which occur in patients with ESRD also affect drug disposition. Anesthesiologists should therefore be knowledgeable about the changes in the metabolism of each drug administered in the perioperative period. The distribution and clearance of the short acting benzodiazepine midazolam remains relatively unchanged making it a drug of choice for anxiolysis in this group of patients.

\section{Induction agents}

The pharmacokinetics and pharmacodynamics of the hypnotic Propofol are unchanged in ESRD patients. Propofol is mainly metabolized in the liver and its metabolites do not have pharmacological activity. Furthermore, it has been shown to be safe for the induction and maintenance of anesthesia in patients with renal failure. Infusion dose requirements have also been found to be similar in ESRD patients and patients with normal renal function although shorter emergence times have been noted in ESRD patients when compared with patients with normal renal function. Thiopental is another inducing agent that 
is almost entirely metabolized in the liver. Its breakdown products are excreted by the kidneys and the gastrointestinal tract. Traces are excreted unchanged in the urine. No permanent effects of this agent on kidney function have been recorded.

\section{Neuromuscular blocking agents}

Succinylcholine is frequently used in general anesthesia to facilitate tracheal intubation because of its rapid onset and brief duration of action. It may however increase serum potassium concentration, which can result in cardiac arrhythmias and even cardiac arrest; especially in patients with ESRD. Therefore, succinylcholine should be used with caution in patients with ESRD. Long-acting nondepolarizing neuromuscular blocking agents are largely eliminated by renal excretion and the effects of long-acting non-depolarizing neuromuscular blocking agents may be significantly prolonged in patients with ESRD, leading to a high rate of residual blockade at the end of surgery. Therefore, in patients with ESRD, muscle relaxants that are not primarily dependent on renal function should be used for general anesthesia. Cisatracurium is intermediate acting musclerelaxant. It is metabolized through Hofmann elimination and produces a metabolite, Laudanosine, which is partially eliminated through the kidneys hence, has a slightly prolonged elimination halflife in patients with renal failure. Overall, the duration of action of cisatracurium is slightly prolonged in renal disease.

Other muscle relaxants such as vecuronium and rocuronium are also eliminated relatively independent of kidney function. Both drugs are mainly metabolized by the liver, but have metabolites that are excreted by the kidney and liver. The duration of action of vecuronium and rocuronium in patients with renal failure has been reported to be slightly prolonged and a cumulative effect has been noted with repetitive administration. Cis atracurium is the preferred muscle relaxant in patients with ESRD.

\section{Opioids}

Perioperative analgesics should be administered with care in ESRD patients undergoing transplantation given that these agents or their active metabolites depend on renal excretion and may accumulate. The effect of morphine is prolonged in patients with chronic renal failure as a result of the accumulation of its active metabolite morphine-6-glucuronide. Similarly, the administration of high or repeated doses of meperidine in these patients may lead to seizures due to the accumulation of its metabolite, normeperidine. The elimination of oxycodone is also impaired in ESRD patients undergoing renal transplant. Importantly, the pharmacokinetics of fentanyl, alfentanil, sufentanil are not altered in chronic renal failure, because the metabolites are inactive and are unlikely to contribute to the opioid effect even if they do accumulate. Remifentanil, a very short acting opioid is metabolized in the peripheral tissues by an esterase enzyme and requires no change in dosing compared to patients with normal renal function. However, total drug clearance and the volume of distribution may be significantly increased.

\section{Inhalational agents}

All potent inhalational agents cause a decrease in the renal blood flow and glomerular filtration rate in proportion to the dose. Many also release fluoride as a byproduct and fluoride has been linked to high output renal failure. Although fluoride is a major metabolite of isoflurane, the extent of its metabolism is very small such that the amount of fluoride produced is unlikely to cause renal damage. It can be used in anesthesia for renal transplantation. Another inhaled agent, Sevoflurane, is metabolized to compound A by carbon dioxide absorbents used in standard anesthesia machines. When sevoflurane is used with lower fresh gas flows, compound A can accumulate within the anesthesia machine gas circuit. Compound A has been shown to be nephrotoxic in rats but has not been reported to increase renal dysfunction in humans with pre-existing renal disease. In fact, Conzen et al. reported that low-flow sevoflurane anesthesia was safe and did not alter kidney function in patients with pre-existing renal disease. Hence, low-flow sevoflurane anesthesia can be safely used in renal transplant recipients. Similarly, desflurane can also be used in patients with renal dysfunction and no deterioration in renal function has been found in patients with pre-existing renal disease who were administered desflurane.

\section{Anesthetic management}

Kidney transplantation is usually performed under general endotracheal anesthesia although they can be done under spinal anesthesia in rare cases. In fact, early kidney transplants were done under spinal anesthesia and some centers in the US still perform neuraxial anesthesia for kidney transplantation. General endotracheal anesthesia provides stable hemodynamics, excellent muscle relaxation, and predictable depth of anesthesia. Combined techniques using GETA and epidural analgesia can also be employed. Hemodynamics and renal function have not been reported to differ significantly between techniques. Adequate monitoring is essential in patients undergoing kidney transplantation. Standard ASA monitors may be sufficient if the patient has no other co morbidities except ESRD. Most patients, however, will benefit from CVP monitoring and possibly invasive arterial blood pressure monitoring given the expected large swings in hemodynamic parameters during surgery. Patients with severe coronary artery disease, left ventricular dysfunction, valvular abnormalities, or pulmonary hypertension may also benefit from advanced intraoperative monitoring such as pulmonary capillary wedge pressure monitoring or intraoperative transesophageal echocardiography. Aggressive volume expansion to a target CVP of $10-15 \mathrm{mmHg}$ is optimal. This volume expansion is associated with increased renal blood flow and improved graft function.

CVP invariably declines in the late intraoperative and immediate postoperative period despite positive fluid balance and vigorous fluid resuscitation. The etiology is unclear but may be due to increased vascular permeability and alterations in vascular tone accompanying surgery. Fluid redistribution in different compartments as a result of preexisting vascular permeability may also be responsible for the decrease in CVP. Graft acute tubular necrosis has been reported to be lower in patients who are vigorously hydrated. Ferris et al. found no correlation between the decrease in CVP and fluid balance although the fluid balance of recipients correlated strongly with immediate post-operative graft function. The maximum decrease in CVP values occurred between the operating room and intensive care unit. Other agents used to promote renal blood flow such as dopamine, dobutamine and fenoldopam) as well as osmotic and loop diuretics (mannitol and furosemide) have been proposed as aiding kidney function after reperfusion. Of these, only mannitol has been shown to decrease the incidence of acute tubular necrosis. Two meta-analyses have shown a negative effect of dopamine on renal function in acute renal failure. In contrast, dobutamine can be used as a positive inotrope for patients with a low cardiac output. Normotension or slight hypertension should be maintained to help graft function, but one should be weary of using alpha agonist, as the vasoconstriction may interfere with renal perfusion. The overall anesthetic goal for the newly transplanted 
kidney is to maintain intravascular volume and avoid decreased perfusion to the new kidney. Normal saline is the intra-operative intravenous fluid of choice in renal transplant recipients. Potassiumcontaining fluids, such as lactated Ringer's solution, are avoided in order to minimize the risk of development of hyperkalemia. Metabolic acidosis may develop following the administration of large volumes of normal saline. The observed acidosis likely due to the chloride infused. The resulting hyperchloremic metabolic acidosis can further lead to extracellular potassium shift, and hyperkalemia. Nevertheless, normal saline is still the fluid of choice in patients undergoing transplantation.

Colloids may be considered in recipients with severe intravascular volume deficits who require high-volume resuscitation. Albumin, a normal endogenous colloid with a wide safety margin is recommended with large fluid deficit. Synthetic colloids that have widely replaced albumin in clinical practice; dextrans, gelatins and solutions of hetastarch; do not seem to be preferable to albumin. Colloids have been reported to adversely affect renal function and may increase bleeding complications. The high potassium and calcium content of gelatins renders them inapplicable for perioperative care during renal transplantation. Dextran solutions have been associated with severe hypersensitivity reactions and coagulation problems. Hydroxyethyl starch (HES), although a synthetic colloid is an alternative to Albumin. HES are a medium molecular weight solution and have the lowest in vivo molecular weight above the threshold for renal elimination. It is also easily degradable. A study concluded that HES given at a maximum dosage of $15 \mathrm{ml} / \mathrm{kg} /$ day to the donor had no detrimental effect on renal function in the graft recipient. Sufficient amounts of crystalloids should, however, be infused along with HES.

\section{Postoperative care}

Most newly transplanted patients can have their neuromuscular blockade reversed and their trachea extubated at the end of surgery. Many patients do not require intensive care unit admission. In fact, most patients are cared for in the medical-surgical floor. Regardless of postoperative ward destination, intravascular volume status and urine output should be monitored closely. These patients need close monitoring in a specialized ward. Sudden decrease in urine output may warrant surgical re-exploration. Transplanted patients requiring prolonged mechanical ventilation postoperatively, have a worse outcome compared with patients who were extubated at the end of surgery. Post-operative pain relief is essential after renal transplantation, as inadequately controlled pain can lead to agitation, tachycardia, hypertension and an increased risk of pulmonary complications. Postoperative pain can either be managed with epidural analgesia if available or by patient-controlled analgesia. Morphine, fentanyl or hydromorphone delivered by patient-controlled analgesia can provide sufficient pain relief. Epidural analgesia provides superior analgesia but may occasionally result in hypotension and decreased renal perfusion further complicating graft survival. Patient controlled analgesia while less effective for pain control is less likely to cause hypotension. The use of non-steroidal anti-inflammatory drugs (NSAIDs) should be avoided. The use of high-dose NSAIDs in patients with chronic kidney disease has been shown to increase the risk of rapid progression of the disease in the elderly age group. Cyclo-oxygenase- 2 enzyme inhibitors have also been reported to be nephrotoxic in transplant recipients and in patients with impaired renal function.

\section{Immunosuppressive therapy}

Immunosuppression varies by center and is usually protocol based.
Regimens can be high dose conventional regimen or an antibody induction regimen. The conventional regimen consists of a calcineurin inhibitor (cyclosporine or tacrolimus), a corticosteroid, and an antimetabolite (mycophenalte mofetil or azthoprine). The antibody induction regimen, which has shown better graft outcomes, uses lower doses of the conventional medications with the addition of an antibody directed at T-cells antigens: anti-lymphocyte antibodies (i.e. Thymoglobulin, alemtumzumab, OKT3) or interleukin-2 receptors antagonist: Basiliximab (trade name Simulect) or Daclizumab (trade name Zenapax). The antibody induction regimen has been shown to result in better graft outcomes. Induction of immunosuppression usually begins with the corticosteroid and an antilymphocyte administered shortly before reperfusion of the renal graft.

\section{Methods}

In this retrospective study we reviewed medical records of 134 cases of living kidney transplants conducted from December 2000April 2005 At Ahmed Gasim hospital.

The hospital policy all drugs used and event that occur perioperative were recorded manually and a copy of the preoperatively were recorded manually and copy of the preoperative assessment and anesthesia notes written by the concerned consultant anesthesiologist were preserved. We noted age, sex, type of transplant, cause of CKD, preoperative preoperative preparations and investigations, details of anesthesia management and monitoring and the outcome were also recorded and entered into an electronic database. All Live donors nephrectomies; were conducted under general anaesthesia with controlled ventilation. Our study through focused on management of the recipients of renal transplant.

\section{Results}

The average hospital stay of the donor is 3 days, the recipient stay is variable 2-3 weeks To date we have done 134 transplants- 28 female recipients, and 106 males. The recipients age is $8-65$ years.

Those less than 40 years of age constitute $57.5 \%$ of the total number of recipients.

Less than 50 years constitute $77.6 \%$ there are only 6 unrelated (or emotionally related) donors. $4.5 \%$.

Paediatric recipients are only 6 children $4.5 \%$ of the total.

One year patient survival $92 \%$, One year graft survival $84.6 \%$ (Figure 1-3)

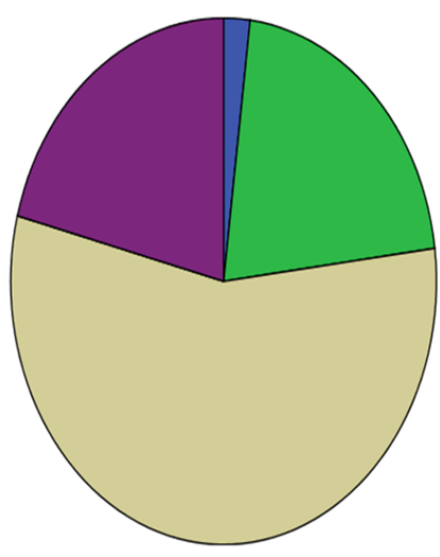

$\square$ less than 5 ol

प5.791

7.1091

I Hemoglobin level before surgery.

$\mathrm{HB}$ s Ag. 


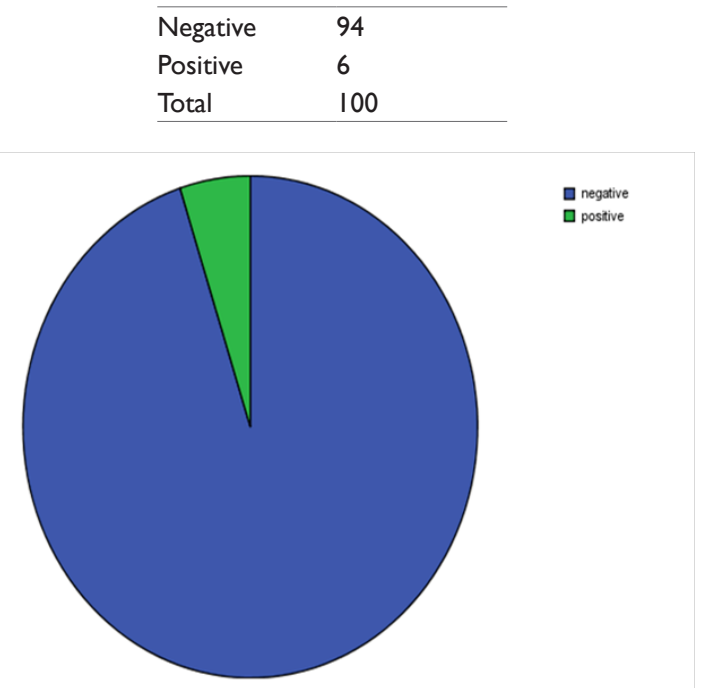

Figure $2 \mathrm{HCV} \operatorname{lgG}$ before surgery. CMV IgG.

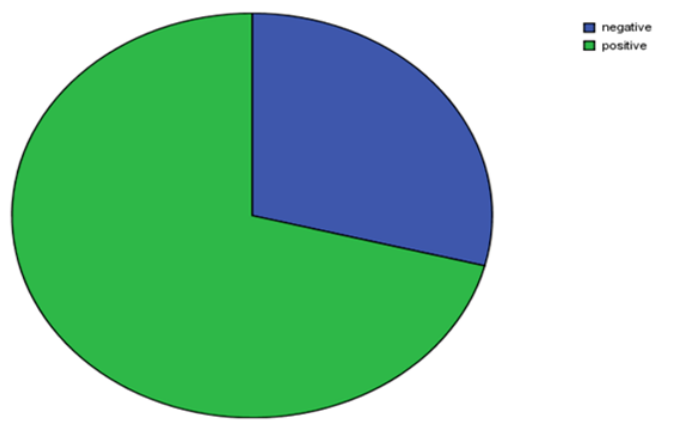

Figure $3 \mathrm{CMV} \lg \mathrm{G}$ before Surgery.

Hypertension

\begin{tabular}{ll}
\hline Hypertension & Percentage \\
\hline Yes & $55 \%$ \\
No & $45 \%$ \\
Total & $100 \%$ \\
\hline
\end{tabular}

\section{Our anesthetic protocol of management}

a. Preoperative evaluation and preparation.

b. Drugs - review.

c. Immunosayressants drugs, triple of prednisolone, cyclosporine (A) and azathioprine. Since February, 2004 the regimen consisted of tacrolimus and azathioprine.

\section{Anesthesia management}

I. General anaesthesia was the technique of choice in most 134 of cases.

II. Epidural anaesthesia with intermittent intravenous sedation was used in 4 cases and outcome was good.

III. At the study institution all the living transplants were done electively.

IV. Hemodialysis was performed in almost all recipients within 24 hours before surgery to reduce the risk of volume overload, hyperkalemia, and excessive bleeding.
V. Peripheral intravenous access was secured in the hand opposite to the functioning fistula and induction of anesthesia was done with propofol $(2 \mathrm{mg} \mathrm{kg})$ neuromuscular blockade was maintained with either atracurium $(0,6 \mathrm{mg} . \mathrm{kg})$ or vecuronium $(0,1 \mathrm{mg} \mathrm{kg})$ all patients were intubated and ventilated.

VI. Anesthesia was maintained with $40 \%$ Nitrous oxide in oxygen supplement with $1-2 \%$ isoflurane, with fresh gas flow of $21 / \mathrm{min}$.

VII. Analgesia was maintained with fentanyl 2-5 mcg kg or morphine $0.1 \mathrm{mg} \mathrm{kg}$.

VIII.Intraoperative monitoring included heart rate, non-invasive blood pressure, oxygen saturation, ends tidal $\mathrm{co} 2$ and electrocardiogram in all patients. Central venous line was placed in right or left internal jugular vein depending upon the presence of dialysis catheter. Average duration of surgery was $5.5 \mathrm{hrs}$ and during this period intra venous fluid administered was normal saline-based crystalloid or colloid, intraopertively $80 \%$ of patients received injection furosemide average 1 to $1.5 \mathrm{mg} / \mathrm{kg}$.

IX. Hemodynamic parameters were recorded on hourly basis from the intraoperative charting

$\mathrm{X}$. Hemodynamic parameters were recorded on hourly basis form the intraoperative dopamine infusion at 2-5 microgram $/ \mathrm{kg}$ was used in 10 patients, 3 patients had intraoperative arrhythmias in the form of premature ventricular contractions.

XI. At the end of the surgery, neuromuscular blockade was reversed with neostigmine $0.05 \mathrm{mg} / \mathrm{kg}$ and glycopyrrolate $8 \mathrm{mcg} / \mathrm{kg}$ intravenously. Most of the patient were extubated immediately postoperatively though recovery was delayed in 4 patients and 6 patient had shouted blood pressure, Hyperkalemia intra operative $0.75 \%$ (Table)

\section{Reversal agents}

Neostigmine: Renal excretion is the principal route of elimination for it, the half live of it are prolonged at least as much as any of muscle relaxants.

\section{Postoperative care:}

a. Were transferred to post kidney transplant care unit, rescue analgesia was provided with fentanyl $2-5 \mathrm{mcg} \mathrm{kg}$ or morphine $0.1 \mathrm{mg} \mathrm{kg}$.

b. Rejection $15.6 \%$, Deep venous thrombosis $4.5 \%$ Pneumonia,

c. Post-transplant diabetes mellitus $6.7 \%$. Post-transplant diabetes mellitus was noticed more with tacrolimus than with cyclosporine,

d. Herpes zoster 3\%, severe cyclosporine nephrotoxicity $0.75 \%$.

e. Discontinuing cyclosporine, the patient restored his normal renal function. Mild nephrotoxicity is common and reversible, Molluscum contagiosum $0.75 \%$.

f. Bone marrow suppression - leucopenia, thrombocytopenia and anemia, Kaposi sarcoma 1.5\%, Septicemia 2.4\%, gastrointestinal symptoms - diarrhea, Oral candidiasis, Esophageal candiasis, Septicemia, Cerebral abscess $1(0.75 \%)$ on discontinuation of steroids, this patient did well. The neurosurgeon was reluctant to operate treated conservatively, Tuberculosis $0.75 \%$.

Surgical complications: Hematoma 4.5\%, Urine leak 2.2\%, Lymphocele $0.75 \%$, Renal vein thrombosis $0.75 \%$, Renal artery thrombosis 1.(0.75\%), Wound infection 3\%, Graft avulsion 1(0.75\%). 


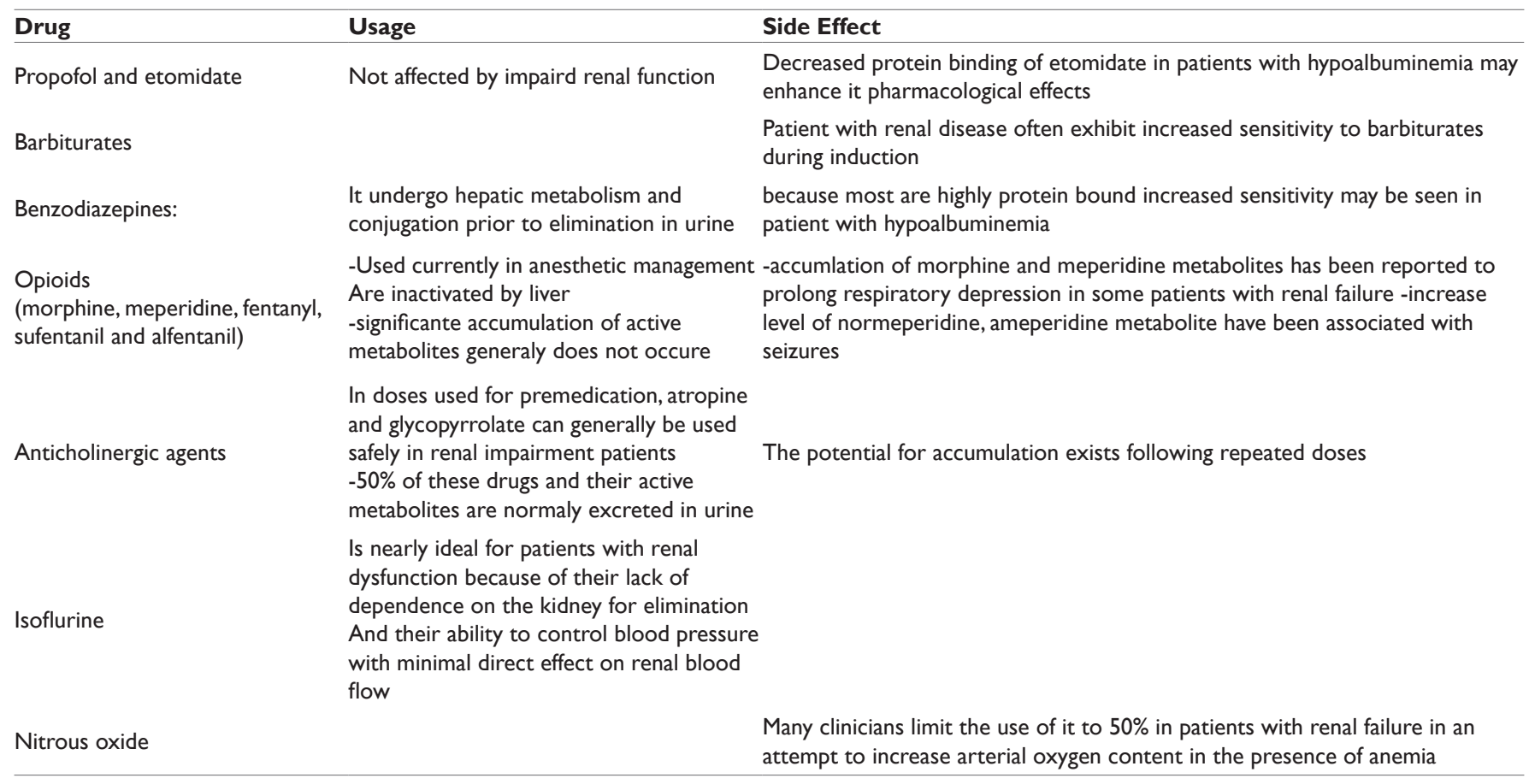

\section{Muscle Relaxants}

\begin{tabular}{|c|c|c|}
\hline drug & usage & Sid effect \\
\hline succinylcholine & $\begin{array}{l}\text { Safely used in the presence of renal failure provided the } \\
\text { serum potassium concentration is known to be les than } 5 \\
\mathrm{mEq} / \mathrm{L} \text { at time of induction }\end{array}$ & $\begin{array}{l}\text { Although decreased pseudo cholinesterase levels have } \\
\text { been reported in a few uremic patients following dialysis, } \\
\text { significant prolongation } \\
\text { Of neuromuscular blockade is rarely seen }\end{array}$ \\
\hline $\begin{array}{l}\text { Cisatracurium, } \\
\text { atracurium }\end{array}$ & $\begin{array}{l}\text { May be the drug of choice for muscle relaxation in patients } \\
\text { with renal failure,they } \\
\text { Are degraded in plasma by enzymatic ester hydrolysis and } \\
\text { nonenzymatic Hofman elimination }\end{array}$ & \\
\hline $\begin{array}{l}\text { Vecuronium, } \\
\text { rocuronium }\end{array}$ & $\begin{array}{l}\text { The elimination of Vecuronium is primarily hepatic but up to } \\
20 \% \text { eliminate in urine. } \\
\text { Rocuronium primarily undergoes hepatic elimination }\end{array}$ & $\begin{array}{l}\text {-The effect of large dose of vecuronium }(>0.1 \mathrm{mg} / \mathrm{kg} \text { ) are } \\
\text { only modestly prolonged in patient with renal insufficiently. } \\
\text {-Rocuronium prolongation of action has been reported in } \\
\text { sever renal disease }\end{array}$ \\
\hline
\end{tabular}

Quality of life: All recipients were off work prior to transplantation, and all of them are back to work post-transplant. They are leading an active life style. Those who were single got married. Ladies with primary infertility fell pregnant, and those with secondary infertility restored their fertility and got healthy babies. Some patients who were working in Saudi Arabia and the Gulf States came home, got transplanted and returned to work abroad. One recipient established a charity organization to help patients with end stage renal failure who are not getting a living related donor by providing a living unrelated donor for them. The quality of life has to be seen to be appreciated. The recipients are discharged when the serum creatinine is normal and no complications. Follow up in the OPD weekly for one month, fortnightly for another month and monthly. Thereafter, there is open access to the transplant ward at any time.

\section{Discussion}

Kidney transplantation is the treatment of choice for patients with end-stage renal disease. 2 It is more cost effective than maintenance dialysis and usually provides the patient with better quality of life.

Any surgical procedure, in patients with CKD has a significant increase in the perioperative morbidity and mortality. Preoperative work-up and intraoperative management of patients with end-stage organ disease are certainly among the most difficult and challenging areas in anaesthesia.

Understanding the myriad alterations in physiology and function, both locally and systemically, is critical to providing safe and successful perioperative management. Co-morbid disease is common and frequently severe in these patients. This audit shows that the renal transplantation programme at this institute has been successful. Factors responsible for good outcome are proper planning and team efforts by all concerned in the transplant team in addition to good preoperative preparation of the patient. Preoperative control of the systemic effects of CKD in recipients, well controlled intraoperative hemodynamics, and good postoperative medical care resulted in success of the transplant program.

Fluid management remains a controversial subject in perioperative medicine and organ transplantation. Recent advances in the understanding of pharmacokinetic and pharmacodynamics profiles of fluids, as well as transplantation physiology and pathophysiology, can guide us in new approaches to common problems. Fluid therapy in transplant medicine is usually best practiced using goaldirected approaches and balanced electrolyte formulations when 
possible. Crystalloid solutions are usually preferred to correct fluid and electrolyte imbalance. In our study, majority of patients $(87 \%)$ were transfused with normal saline-based crystalloids; as most anaesthesiologists would avoid potassium containing fluids during renal transplantation, with the belief that it may worsen the hyperkalemia in the event of impaired graft function. However in a recent randomized, double blind study comparing Ringer's solution and $0.9 \%$ normal saline during renal transplant the authors have shown those who received Ringer's solution had less hyperkalemia and acidosis. The saline infusion leads to acidosis possibly by dilution of bicarbonate by large volumes of buffer free fluid or the resultant hyperchloremia decreases the strong ion difference with the development of acidosis. The hyperchloremic metabolic acidosis leads to hyperkalemia by shift of potassium into extracellular space. However in this study the average volume of crystalloid infused during the surgery was about 6 liters.

Both crystalloids and colloids have been used for volume replacement. Over the last few decades there has been a shift in practice from using natural colloid to synthetic colloids. There has been some concern regarding the use of hydroxyethyl starch (HES) as osmotic, nephrosis-like lesions were demonstrated in transplanted kidneys retrieved from deceased donor who were transfused with HES200/0.62 7 In our study 44 (12\%) patients received a combination of crystalloids and colloids with the colloid being mainly gelatin based. Some fluids may exert drug effects that could alter organ preservation and reperfusion, while the low molecular weight HES appears to be less toxic in renal transplantation than first suspected, especially when clinicians consider free water requirements in these settings.

Standard ASA monitors were used in all the patients, however in patients with more advanced stages of co-morbid conditions, more extensive monitoring such as CVP monitoring (all patients) or continuous arterial pressure monitoring (15patients) were used. Inotropic support was used in 4 patients. No major rise in blood pressure was seen in these patients. In a large series of renal transplantation by Heino $\mathrm{H}$ and Orko R hypotension (49\%) was a more common finding than hypertension $(26.8 \%)$.

A retrospective study of postoperative respiratory morbidity in 247 patients requiring renal transplantation showed that 7 patients required postoperative controlled ventilation. Long acting nondepolarising relaxants were used in only 65 patients, but all 7 cases of respiratory failure occurred in this group, which suggests that the use of these drugs in anephric patients is potentially hazardous so far as postoperative respiratory insufficiency is concerned.10 In an other study by Avner Sidi and Richard Kaplan, prolonged neuromuscular blockade has been reported in 8 out of 65 patients of renal transplant who had received either vecuronium(4 out of 29) or atracurium.

In our study neuromuscular blockade was maintained with atracurium Persistent neuromuscular blockade with delayed recovery was seen in 7 patients and needed postoperative ventilatory support. In our study only 5 cases were done using continuous epidural anaesthesia with intermittent intravenous sedation. No major complication was reported in either of these cases. There have been reports of renal transplants done under continuous epidural anesthesia with intermittent sedation with intravenous agents by Lauretti, Gabriela Rocha. They also reported frequent respiratory complications and intraoperative rupture of the renal anastomosis due to cough, hiccups and agitation. As an alternative technique, the laryngeal mask airway (LMA) was used to maintain clear upper airways during continuous epidural anesthesia.
Transplant anesthesia is a specialized field, which requires a good understanding of the abnormalities in Patients with renal failure, familiarity with transplant Medicine and expertise in management of these patients. With improvement in anesthetic and surgical techniques as well as immunosuppressive drugs, many patients are being accepted for transplantation who would have been considered unsuitable earlier. Proper patient selection, preoperative patient preparation and intraoperative physiological stability with close association between nephrologist, urosurgeons and anesthesiologists have found a valuable place in the management of our renal transplant patients and has given us good results. Further large scales studies are desired.

\section{Clinical application of the study}

This study concerning with living donors, which make the operation of the transplantation elective so there is always sufficient time to optimization recipient comorbidities in the preoperative period and others recipients general condition like Fluid status, hemodynamic stability (HB,ELECTROLITSES ), fitness of the patient and you should interpretate all these results to choose the best anesthetic agent for every individual patient and close intraoperative monitoring are keys of success in renal transplantation.

\section{Limitation of the study}

The first factor of the limitation is the retrospective of the study and the experience of the study is ten year ago so a lot of advancement in technique and management of renal transplantation has happened. Now in this center (AHMED GASIM) we exceed more than one thousand transplants and we will review all of them. The second factor is that donors for transplantation are close relative living donors, no cadaveric donors at all. ${ }^{1-12}$

\section{Acknowledgments}

None.

\section{Conflicts of interest}

The authors declare there are no conflicts of interest.

\section{Funding}

None.

\section{References}

1. Sprung J, Kapural L, Bourke DL, et al. Anesthesia for kidney transplant surgery. Anesthesiology Clinics of North America. 2000;18(4):919-951.

2. Lemmens HJ. Kidney transplantation: recent developments and recommendations for anesthetic management. Anesthesiol Clin North America. 2004;22(4):651-662.

3. Evans RW, Manninen DL, Garrison LP Jr, et al. The quality of life of patients with end-stage renal disease. $N$ Engl J Med. 1985;312(9):553559.

4. Johnson RJ, Armstrong S, Belger MA, et al. The Outcome of cadaveric renal transplantation in the UK and Eire. Pediatr Transplant. 2002;6(5):367-377.

5. Elshihabi I, Chavers B, Donaldson L, et al. Continuing Improvement in cadaveric graft survival in North American Children. The 1998 Annual report of the North American Pediatric Renal Transplant Cooperative Study. Pediatric Transplantation. 2000;4(3):235-246.

6. O’Malley CM, Frumento RJ, Hardy MA, et al. A randomized Double blind comparision of lactated Ringer's solution and $0.9 \% \mathrm{NaCl}$ during renal transplantation. Anesth Analg. 2006;100(5):1518-1524. 
7. Cittanova ML, Leblanc I, Legendre C, et al. Effect of hydroxy ethyl starch in brain dead kidney donors on renal function in kidney transplant recipients. Lancet. 1996;348(9042):1620-1622.

8. Roche AM, James MF. Fluid therapy in organ transplantation. Curr Opin Organ Transplant. 2007;12(3):281-286.

9. Heino A, Orko R, Rosenberg PH. Anaesthesiological problems in renal transplantation: A retrospective study of 500 transplantations. Acta Anaesthesiologica Scandinavica. 1986;30(7):574-580.
10. Rouse JM, Galley RL, Bevan DR. Prolonged curarisation following renal transplantation. A retrospective study. Anaesthesia. 1977;32(3):247251

11. Sidi A, Kaplan RF, Davis RF. Prolonged neuromuscular blockade and ventilatory failure after renal transplantation and cyclosporine. Can J Anaesth. 1990;37(5):543-548.

12. Lauretti, Rocha G, Garcia, et al. Use of laryngeal mask airway during continuous epidural anesthesia for renal transplant. Rev bras anesthiol. 1996;46:107-113. 